\title{
Glutathione S-transferase polymorphism and prostate cancer
}

In recent years, glutathione $S$-transferase genotype has been extensively studied as a prostate cancer risk factor. Findings have been disappointingly inconsistent. Now, a large meta-analysis by Chinese researchers of 39 case-control studies has refined our understanding of how differences in these carcinogenmetabolizing enzymes influence the likelihood of developing this disease.

Of the three glutathione $S$-transferase loci studied, only polymorphism of GSTM1 was found to be associated with prostate cancer risk. Data from across ethnic groups showed that homozygous deletion of GSTM1, which results in a nonfunctional enzyme, increased the likelihood of developing prostate cancer by about $30 \%$. Polymorphisms of GSTT1 and GSTP1 did not seem to affect disease risk. Subgroup analysis of data from the 7,984 cancer cases and 9,143 controls showed that the increased risk conferred by the GSTM1 null genotype applied to Caucasian and Asian populations, but not to African/African-American men. Lead author Zengnan Mo from The First Affiliated Hospital of Guangxi Medical University notes, however, that "the stability of the results of any meta-analysis is affected by the heterogeneity of studies included, so the interpretation of [these] results should be cautious."

The clinical significance of the GSTM1 polymorphism has not yet been determined. "A large-scale study of thousands of participants involving tissue-specific biochemical and biological characterizations needs to be done to study this in more detail," Mo concludes.

Kathryn Senior

Original article Mo, Z. et al. An updating meta-analysis of the GSTM1, GSTT1, and GSTP1 polymorphisms and prostate cancer: a HuGE review. Prostate $69,662-688$ (2009). 